\title{
Clinical records on computer for ambulatory patients
}

\author{
CHRISTOPHER D. OLSON, DO
}

\begin{abstract}
Computer systems now are
available not only to record patient information but to use that data to help the physician care for patients. Besides maintaining records in a readable form, the computer can provide valuable summaries and access to data from a remote site, increase efficiency of paperwork through avoiding re-entry procedures, and remind the physician to perform preventive, diagnosis-related, and treatment-related procedures. Costs and problems associated with the use of such systems are not unreasonable when compared to the benefits.
\end{abstract}

The computer has become an important instrument for physicians for a variety of purposes. This paper explores the most valuable features of computerized records, and some potential problems and costs. Several software programs are described briefly.

See December DO for articles on computers in medicine.

\section{Features}

Although the ability to read clinical record data is an important feature of computerization, maintainance of information in a usable fashion, avoidance of re-entry of data, and use of data to generate reminders all may be more valuable functions of the electronic record system.

A quick glance at a problem or medication list or, perhaps, a graph of vital signs and critical laboratory data can be extremely useful in emergency situations or at any time to review a patient's history. Unfortunately, these lists often are not kept, not current, or not readily available. The most likely reason is that it is time consuming to rewrite and keep current data that has already been recorded in progress notes. Even if all of these lists and graphs are maintained properly, they often are not available when they are needed (such as in the emergency room or when the patient telephones the physician at home).

A computerized record system can sort data entered in a progress note automatically, can maintain up-to-date problem and medication lists, and even can graph numerical data. It can make these data available with reasonable security to remote sites such as the emergency room or the physician's home. Finally, such data can be used for crosspopulation searches for research or medication recall purposes.

Using data more than once is the key to making a clinical computer system cost effective. This feature requires integration with business systems to be most effective. The diagnosis, for example, may be written in the progress notes, on the patient's billing form, on insurance forms, and, perhaps, in correspondence to a consultant or referring physician. Demographic data are used for business and medical purposes. Also, prescriptions must be recorded on the chart and then rewritten for the pharmacist, who must rewrite the information for the patient. A computer system can, with a few key strokes, help the physician to find the right name, dose, and quantity of the medication, record it, check for interactions, print a prescription (or send it electronically to the pharmacy of the patient's choice), and print instructions for the patient.

Probably the greatest potential for improving quality of care comes from the computer's ability to remind. It has been shown in a study ${ }^{1}$ on airline pilots that reliance on memory in critical situations is a vital error. An electronic record system can remind us to perform preventive medicine procedures (for example, mammography annually for women past age 50), diagnosis-related procedures (for example, electrocardiography annually for patients with a history of myocardial infarction), and treatment-related procedures (for example, potassium determinations quarterly for a patient taking a thiazide drug). Potential drug interactions can be identified automatically on entry of a new medication. McDonald and associates ${ }^{2,3}$ have shown that these actions are taken twice as often when a reminder is provided than when it is not. 


\section{Potential problems and costs}

Common concerns in using the computer for memory jogging are that the recommendations will become out of date or that a particular physician will not agree with them. Medi-Span drug and drug interactions data sets are one example of how to keep data current. These sets, which are used by several systems for prescription writing and interaction checks, are updated quarterly at a reasonable cost. Most systems that provide reminders make them either user-defined or user-modifiable. Any system that appears to recommend patient care likely will come under FDA scrutiny in the near future as a "medical device." 4

Entering and accessing data efficiently and unobstrusively also are significant aspects of computer use. McDonald ${ }^{5}$ notes that "most of the labor problems and costs of establishing a medical record system are on the input side," but that much data potentially can be captured from other sources. Further, there is some concern about the intrusion of the computer into the physician-patient relationship. ${ }^{6}$ To utilize such functions as printing prescriptions and patient information, the physician or nurse must enter some current clinical data, including new medications and diagnoses, while the patient is present. For access to historic data, a paper summary must be printed for each encounter or a computer terminal must be present wherever the physician or nurse sees the patient. Therefore, patients will need to be acclimated to computers in this setting as well.

The cost of a basic computer system will be $\$ 20,000$ to $\$ 100,000$, and additional personnel time probably will be required. However, Lloyd ${ }^{7}$ demonstrated that in the first year of use in his office, computerization using Duchess software generated more income than outgo and also resulted in fewer patient visits. The increased income was produced by performing recommended tests and preventive measures that had been overlooked previously. Also, computer-generated postcard reminders for flu shots can increase compliance to near $90 \%$ (communication, S.C. Lloyd, September 1986) from what it is commonly $20 \% .^{8}$ This mechanism can produce significant income. The potential savings in time by avoiding re-entry of data has been discussed previously.

Improved quality of care should decrease costs for patients by preventing serious illness and the attendant high cost of hospitalization and longterm care. The systems themselves are likely to become more affordable as hardware costs continue to drop, methods of data entry improve, and new technology creates greater efficiency. For example,
TABLE 1. SOURCES FOR SOFTWARE PROGRAMS.

The Consultant Series

Medical Software Consortium

PO Box 76069

St. Peters, MO 63376

(314) 928-7373

Duchess Corporation

900 Elmwood

Columbia, SC 29201

(803) 779-0557

LifeCard International, Inc.

(Subsidiary of Blue Cross and Blue Shield of Maryland)

Nottingham Centre

502 Washington Ave

Suite 300

Towson, MD 21204

(301) 494-4800

Medi-Span

5980 W 71st St

PO Box 68875

Indianapolis, IN 46268-0875

(800) 428-4495

Practice Partner

Physician Micro Systems, Inc.

2033 Sixth Ave

Suite 707

Seattle, WA 98121

(206) $441-8490$

Smart Chart

Ash Medical Systems, Inc.

2701 B Kent Ave

West Lafayette, IN 47906

(317) 463-940

The Medical Record (TMR)

Database, Inc

PO Box 3054

Durham, NC 27705-1054

(919) 493-6969

The Regenstrief Institute for Health Care

$1001 \mathrm{~W}$ Tenth St

Indianapolis, IN 46202

(317) 630-7400

optical storage devices (laser discs) make storage of large volumes of data more affordable. Insurance companies and the government also are showing signs of interest in supporting such systems. One example is LifeCard, which is owned by Blue Cross and Blue Shield of Maryland.

\section{Available software systems}

Of the dozen of computerized record systems available, some are simply modified word processors with few features other than recording of data. Several business systems have begun offering add-on record components. Some of the most sophisticated systems have been developed at universities, these 


\section{For patients with elevated cholesterol}

\section{THS MAY BE THE \\ BETIER ANIIHYPERTENSIVE}

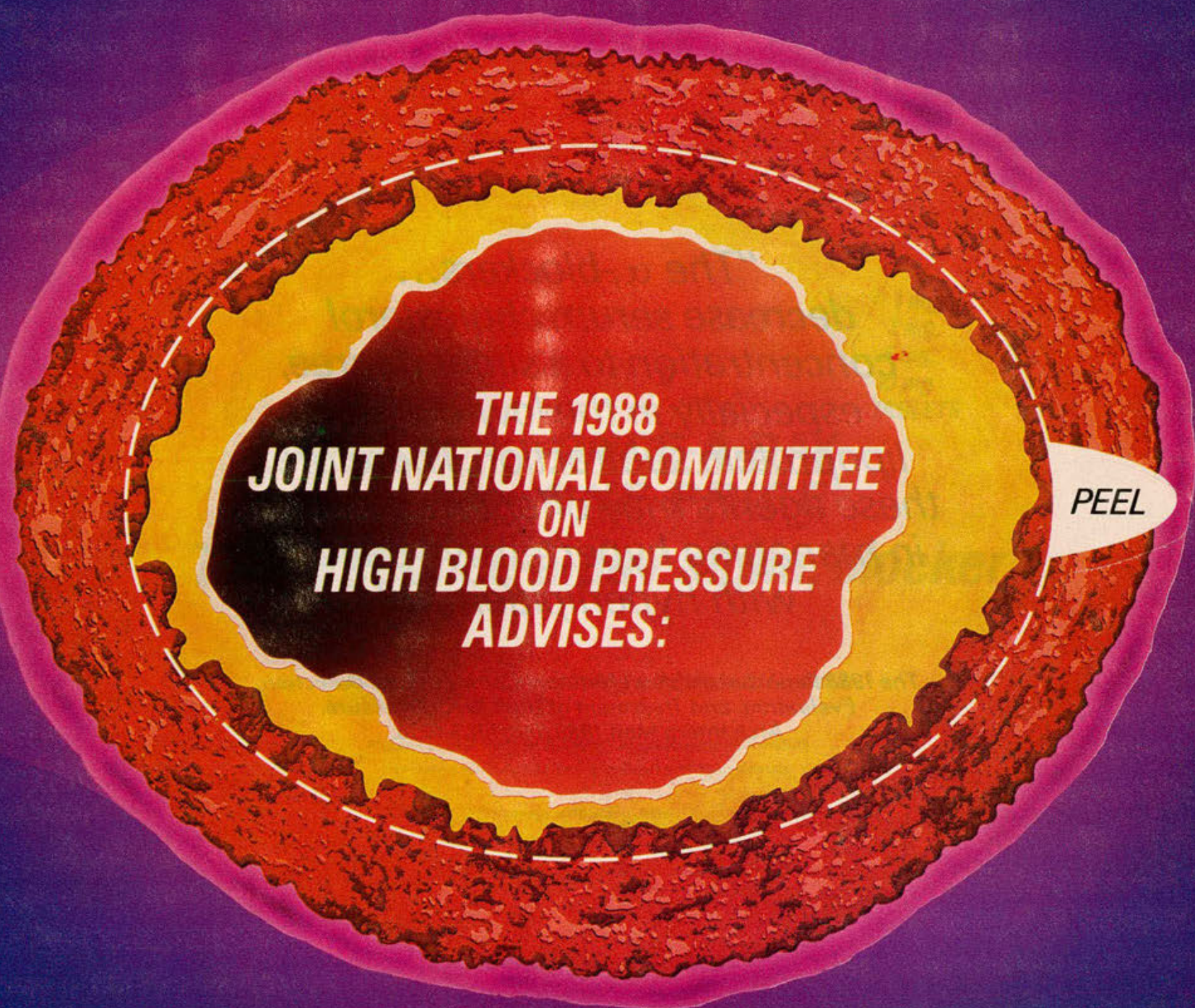




\section{Superior lipids performance in hypertensive patients with elevated cholesterol ${ }^{1.2 *}$}

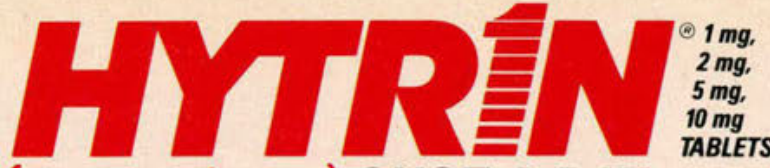

(teraZOSin HCI) ONCE-A-DAY The first once-a-day alpha, blocker

During controlled clinical studies, patients receiving HYTRIN had a small but significant decrease $(-3 \%)$ compared to placebo in total cholesterol and $L D L+V L D L$ cholesterol fraction. ${ }^{1.2}$
Although HDL fraction showed a slight increase from baseline and triglycerides decreased, neither change was significant compared to placebo."

\begin{tabular}{|c|c|c|c|c|c|}
\hline & $\begin{array}{c}\text { Alpha, blockers } \\
(\text { HYTRIN) }\end{array}$ & $\begin{array}{c}A C E \\
\text { inhibitors } \\
\end{array}$ & $\begin{array}{c}\text { Calcium } \\
\text { antagonists }\end{array}$ & Diuretics $^{3}$ & $\begin{array}{c}\text { Beta } \\
\text { blockers }^{3} \\
\end{array}$ \\
\hline Total cholesterol & $\downarrow$ & $\longleftrightarrow$ & $\longleftrightarrow$ & $\mathbf{1}$ & $\longleftrightarrow$ \\
\hline$L D L+V L D L$ cholesterol & $\downarrow$ & $\longleftrightarrow$ & $\longleftrightarrow$ & $\mathbf{T}$ & $\longleftrightarrow 1$ \\
\hline HDL cholesterol & $\Longleftrightarrow 1$ & $\longleftrightarrow$ & $\longleftrightarrow$ & $\longleftrightarrow 1$ & $\downarrow$ \\
\hline Triglycerides & $\longleftrightarrow 1$ & $\longleftrightarrow$ & $\longleftrightarrow$ & 1 & $\uparrow$ \\
\hline
\end{tabular}

\section{HYTRIN ${ }^{*}$}

(terazosin hydrochloride tablets

Brief Summary

CLINICAL PHARMACOLOGY: Pharmacodynamics: Clinical studies of tecazosin used in once-a-day (majority)

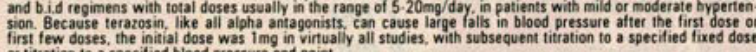
or titration to a specified blood pressure end point.
Blood pressure responses were measured at the end of the dosing interval (usually $24 \mathrm{hrs}$ ) and effects were greatet than placebo the responses in the standing position tended to be somewhat larger, although this was ne irue in all studies. The magnitude of blood pressare responses was similar to prazosin and less than hydrochloroth

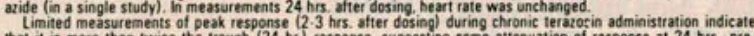

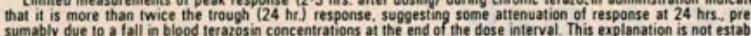
sumably due to a fall in blood terazosin concentrations at the end of the dose interval. This explanation is not estab. dosing With the atsence of an observed dose-response relationship over a range of 5-20-m ie trations fall to the point of providing less than full effect at 24 hirs. A shonter dosing interval or larger dose shoul factory, patients may be tried on a larger dose of bi.d. regimen. The latter should be considered if side effects. The greater BP effect associated with peak plasma concentrations (first few hours after dosing) appears some

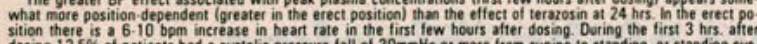

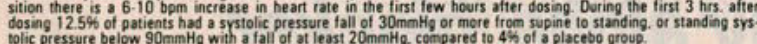
INDICATIONS AND USAGE; Indicated for the treatment of hypertension.

CONTRAINDICATIONS: None known.

WARNINGS: Syncope and "First-dose" Effect: Terazosin, like other alpha-adrenergic blocking agents, can

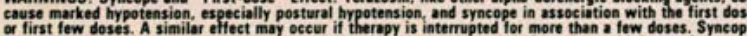
has been reported with other alpha-adrenergic blocking agents in association with rapid dosege increases with heart rates of of $120-160 \mathrm{bpm}$.

To decrease the likelihood of syncope or excessive hypotension, always initiate treatment with a $1 \mathrm{mg}$ dose at bedtime. The $2 \mathrm{mg}, 5 \mathrm{mg}$ and $10 \mathrm{mg}$ tablets are not indicated as initial therepy. Increase dosage injury could resilt if syneope occurs during initiation of therapy.

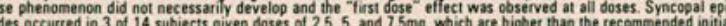
dose. Severe orthostatic hypotension (BP $50 / 0 \mathrm{mming})$ was seen in iwo others and dizziness, tachycardia, and light headedness occurred in most subjects. These adverse effects all occurred within 90 min of dosing 19 of patients, in no case severe or prolonged, and was not necessarily associatted with earily doses. If syncope occurs, place patient in recumbent position and treat supportively. There is evidence that the
orthostatic effect of terazosin is greater, even in chronic use, shortly after dosing. PRECAUTIONS: General: Orthostatic, Mypotension: While syncope is the most severe orthostatic effect of

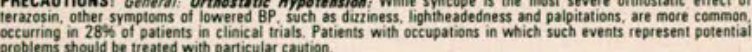
Information for Patients: Make aware of possibility of syncopal and orthostatic symptoms, especially at initiation
of therapy. and to avoid driving or hararoous task for 12 hirs. after the first dose. after a dosage increase, and ther interruption of therapy when treatment is resumed. Caution to avoid situations where injury could resu should sypcope occur during initial therapy. Advise to sit or lit down when symptoms of lowered BP occur and to
rise carefully from a sitting or lying postion. Bothersome diziness. lightheadedness, or palpitations should be reported to physician.
Tell patients that drowsiness or somnolence can occur, reguiring caution in people who must drive or operate Laboratory Tests. Small but statistically, significant decreases in hematocrit, hemoglobin, WBC, total protein and
abumin were observed in clinical trials. The magnitude of decteases did not worsen with time. These findings sug.
gest the possibility of hemodilution. Drug Interactions: in controlled trials, terazosin was added to diuretics, and several beta. adrenergic blockers: no

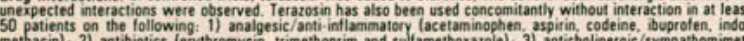

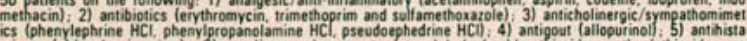

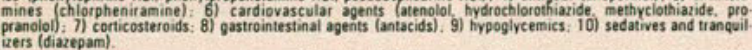

Carcinogenesis, Mutagenesis, Impoairment of fortility: HYTRiN was devoid of mutagenic potential when evaluated
in vivo and in vitro statistically sigiticant increase in benion adrenal medullary tumors of male rats exposed to the $250 \mathrm{mg} / \mathrm{kg}$ dose oncegenic in mice when administered in feed for 2 rrs. at a maximum tolerated dose of $32 \mathrm{mg} / \mathrm{kg} / \mathrm{day}$. assay, of increased total tumer incidence in either species, and of proliferative adrenal lesions in female rats. sug. been associated with these tumors in male rats without supporting evidence for carcinogenicily in man. Iemale rats were administered oral doses of 8,30 and $120 \mathrm{mg} / \mathrm{kg} / \mathrm{day}$. Four of $20 \mathrm{male}$ rats given $30 \mathrm{mg} / \mathrm{kg}$ and of 19 male rats given $120 \mathrm{mg} / \mathrm{kg}$ failed to sire a litter. Testicular weights and morphology were unaffected. Vagina lation was reported between sperm count and subsequent pregnancy.
Oral use for 1 or 2 yrs. elicited a statistically significant increase in testicular atrophy in rats exposed to 40 and $250 \mathrm{mg} / \mathrm{kg} /$ day, but not in rats exposed $108 \mathrm{mg} / \mathrm{kg} /$ day $(>20 \times \max$, recommended human dose). Testicular atro. hot atter $1 \mathrm{y}$. when dosed with $20 \mathrm{mg} / \mathrm{kg} / \mathrm{day}$. This lesion has also been seen with Minipress' Pregnancy: Teratogenic effects: Pregnancy Category C. There are no adequate and well-controlled studies in preg.
nanf women and the saftety of terazosin in pregnancy has not been established. HYTRIIN is not recommended during pregnancy uniess potential benefit justifies pottential risk to mother and fetus. Nonteratogenic effects In a peric and post-natal development study in rats, significantly more pups died in the
group dosed with $120 \mathrm{mg} / \mathrm{kg} / \mathrm{day}(>300 \mathrm{x} \max$. recommended human dose) than in the control group during the Nursing Mothers: It is not known whether terazosin is excreted in breast milk: therefore, exercise caution when

ADVERSE REACTIONS: The prevalence of adverse reactions has been ascertained from 14 placebo-controlled ac in combination with other antimypentensive agents, at doses ranging from 1 to $40 \mathrm{~mm}$. All adverse events reporte during these studies were recorded as adverse fictions. Adverse events wherit the prevalence rate in the teraros group was at least $5 \%$, where the prevalence rate for the terazosing group was at least $2 \%$ and was greater than

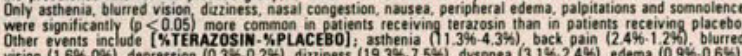

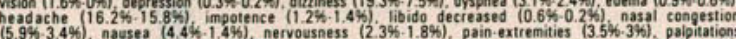

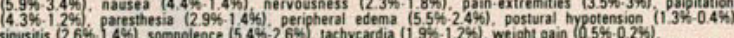

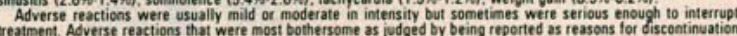
treatment. Adverse reactions that were most bothersome as judged by being reported as reasons for discontinuation

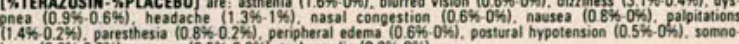

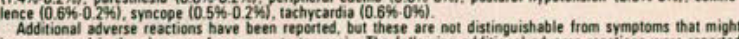
have oceurred in the absence of exposure to terazosin. The tollowing additional adverse reactions were reforited nat pain, abnormal vision, anxiety, arritythmia, arthralgia. arthritis, bronchitis, chest gain, cold symptoms, conjunc.

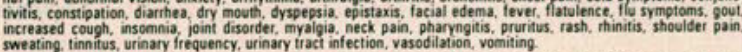
DOSAGE AND ADMINISTRATION: Dose and dose interval ( 12 or 24 hrs) should be adjusted according to BP ne

Initial Dose: $1 \mathrm{mg}$ at bedtime. Observe the initial dosing regimen strictly to minimize potential for severe hypoten Subseguent Doses: Slowly increase dose to achieve desired BP ressonse. Usual dose range is $1 \mathrm{mg}$ to $5 \mathrm{mg}$ once

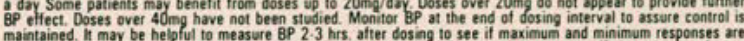
maintained. II mar be helpfult to measure BP 2.3 hrs after dos ing to see it maximum and minimum responses are

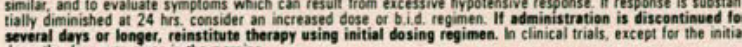
dose, the dose was given in the morning.

Use With Other Drugs: Caution should be observed when terazosin is administered concomitantly with other an

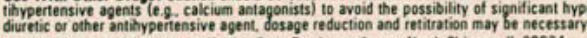
Revised: Sept, 1988 abbott Health Care Products, Inc. North Chicago, II. 60064

References: 1. HVTRIN Product Information, Abbott Laboratories. 2. Deger G: Effect of terazosin on serum lipids. Am J Med 1986;80(suppl 5B):82-85. 3. Dzau VJ: Evolution of the clinical management of hypertension: Emerging role of "specific" vasodilators as initial therapy. Am J Med 1987:82(suppl 1A):36-43. 4. Weinberger MH: The effects of antihypertensive therapy on lipids. Cardiovasc Med 1986;11:10-11. 5. Ames RP: The effects of antihypertensive drugs on serum lipids and lipoproteins. II. Non-diuretic drugs. Drugs 1986;32:335-357. 


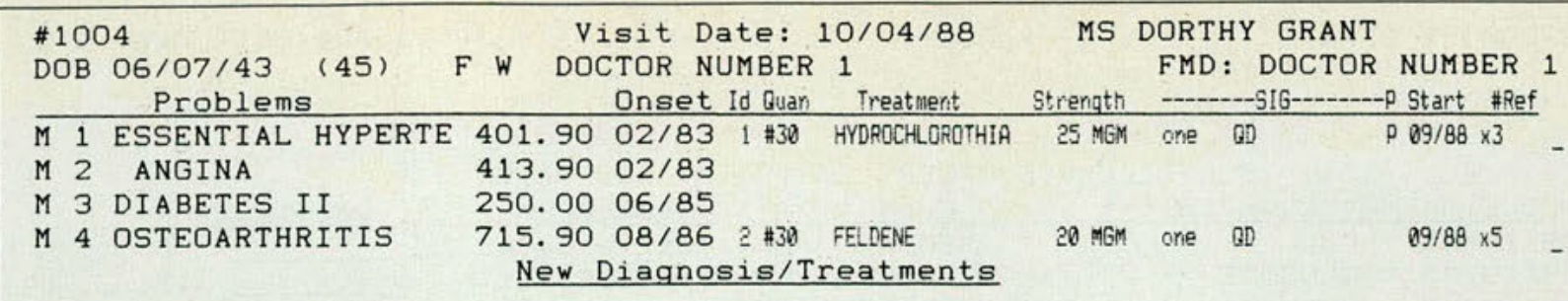

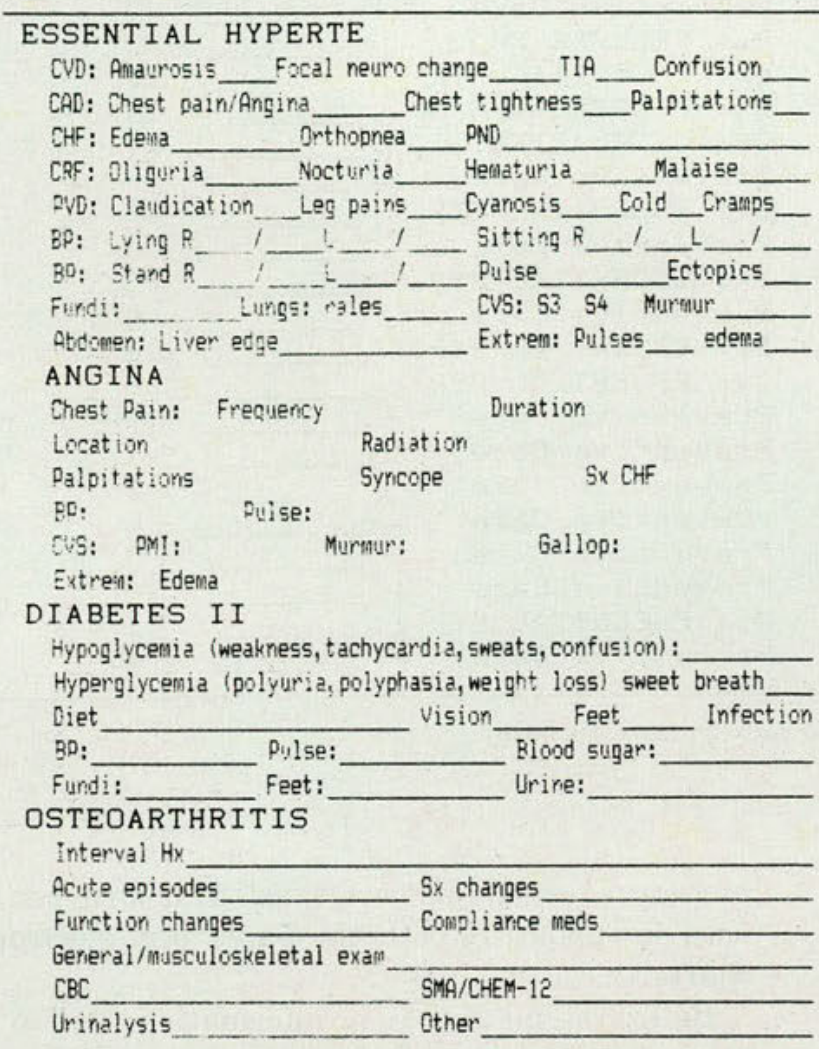

Fig 1. Patient encounter form Duchess software.

make the greatest use of computer intelligence for reminders to perform certain tests or procedures. Some systems incorporate artificial intelligence to help make decisions.

These larger systems may be too large to consider for private practice at this time but have the potential for providing the framework for smaller systems. These latter systems, particularly those from Digital Equipment Corporation, mostly are run on minicomputers. Most of the commercially available systems run on IBM AT or IBM-compatible hardware. Table 1 lists the sources for each software program discussed.

\section{LifeCard}

The LifeCard is a credit-card-sized, permanent personal medical record that the patient carries so that information is readily available wherever the patient may be. The system requires a reader/writer and an IBM personal computer or IBM-compatible equipment for input and output.

\section{Consultant Series, Smart Chart}

The Consultant Series and Smart Chart software do not at present integrate with business systems. Each has the capability of storing progress notes, maintaining problem lists, medication lists, and basic numerical data. Smart Chart uses a laptop computer to record all of these data on floppy disks while the physician is with the patient. Both can perform prescription writing and provide drug interaction information. Neither produces any reminders but each has some capacity for user-defined recall. Smart Chart costs about $\$ 1,500$. The Consult- 
SURVEILLANCE ORDERS SORTED BY DUE DATE

\begin{tabular}{|c|c|c|c|c|}
\hline DUE & Exam/Test & Freq & Reason & Delete \\
\hline *09/19/85 & $\mathrm{CBC}$ & SEMIAN & Preventive Care & \\
\hline *09/19/85 & GLUCOSE & SEMIAN & DX: DIABETES II & \\
\hline *09/19/85 & HGBA $1 \mathrm{C}$ & SEMIAN & DX: DIABETES II & \\
\hline *09/19/85 & OCCULT BLOOD & SEMIAN & Preventive Care & \\
\hline *02/23/86 & BUN & ANNUAL & RX: FELDENE & \\
\hline$* 02 / 23 / 86$ & CHEST XRAY: PA & ANNUAL & DX: ESSENTIAL HYP & \\
\hline *03/23/86 & CREATININE & ANNUAL & Rx: FELDENE & \\
\hline *03/23/86 & SED RATE (WESTE & ANNUAL & DX: OSTEOARTHRITI & \\
\hline *03/23/86 & SMA 12 & ANNUAL & Preventive Care & \\
\hline *03/23/86 & STRESS EKG & ANNUAL & DX: ESSENTIAL HYP & \\
\hline *03/23/87 & LOV: LIMITED OV & 2 YRS & Preventive Care & \\
\hline$* 03 / 22 / 88$ & ECHOCARDIOGRAM & 3 YRS & DX: ESSENTIAL HYP & \\
\hline$* 03 / 22 / 88$ & TONOMETRY & 3 YRS & Preventive Care & \\
\hline *09/11/88 & UR INALYSIS & SEMIAN & DX: ESSENTIAL HYP & \\
\hline $03 / 15 / 89$ & COMPREHENSIVE E & ANNUAL & Dx: ESSENTIAL HYP & \\
\hline $03 / 15 / 89$ & EKG & ANNUAL & Preventive Care & \\
\hline $03 / 15 / 89$ & PAP SMEAR & ANNUAL & Preventive Care & \\
\hline $02 / 22 / 90$ & AUDIOM & 5 YRS & Preventive Care & \\
\hline $03 / 22 / 90$ & MAMMOGRAM & 5 YRS & Preventive Care & \\
\hline $03 / 22 / 90$ & PPD SKIN TEST & 5 YRS & Preventive Care & \\
\hline $03 / 22 / 90$ & SEROLOGY & 5 YRS & Preventive Care & \\
\hline $03 / 15 / 91$ & T 4 & 3 YRS & DX: ESSENTIAL HYP & \\
\hline $03 / 21 / 95$ & TETANUS TOXOID & 10 YRS & Preventive Care & \\
\hline
\end{tabular}

Fig 2. Surveillance order form Duchess software.

ant Medical Record System costs $\$ 1,995$. The Consultant will soon have its own integrated business package (at additional cost).

\section{Practice Partner, Duchess}

The Practice Partner and Duchess are AT-based software systems (Duchess is also available for DEC hardware) that have fully integrated clinical and business functions. Both maintain demographics, problem lists, and medication lists and use these data for a variety of purposes. Duchess can perform prescription writing and give drug interaction information.

The Practice Partner will soon be able to do the same. The latter maintains more information, including notes as well as laboratory and other studies data, and produces flow charts. However, its reminder system essentially is limited to prevention. Duchess, on the other hand, concentrates on reminders based on demographics, problems, and medications. It produces a unique paper "encounter form" for each patient visit (Figs 1 and 2). This form lists problems and related treatment, suggests subjective and objective data to be addressed relative to listed problems, and reminds when preventive and other diagnosis- and treatment-related procedures should be performed. This form can be used for updating both the paper and electronic charts.

Both systems can keep information files to be printed for patients when a new drug or different problem is encountered. The cost of The Practice Partner software is approximately $\$ 7,000$. Duchess is sold on a basis of percent of increased practice volume.

\section{The Medical Record, Regenstrief Institute System} The Medical Record (TMR) (VAX Minicomputer, Digital Equipment Corporation) and the system at the Regenstrief Institute (Regenstrief medical records) are highly sophisticated systems with all of the capabilities described above. Each requires a significant investment in hardware. The Regenstrief system is not available commercially.

As with any facet of computers, changes in medical systems may occur daily. New systems and new features become available. The government or other third parties may develop a universal record system. Improvements in clarity and ease of access to data will make systems more usable. Improved integration between functions will enhance economy and greater use of reminders will improve 
quality of care. Health-care providers and patients will come to accept the inclusion as opposed to intrusion of the computer.

\section{Comments}

Clinical record systems on computer are to current paper medical record systems as the present systems are to $3 \times 5$ index cards of the past. The most pressing reason for using computers for medical records is to improve quality of care. With a computer to manage data, there is greater and clearer access to information. By reusing data, and, thus, avoiding re-entry, efficiency improves. Further, efficient and clear record systems have become a necessity for medicolegal reasons. Undeniable costs and potential problems are associated with such systems, but these are not insurmountable and are outweighed by the advantages.

1. Drinkwater BL: Performance of civil aviation pilots under conditions of sensory input overload. Aerosp Med 1967;38:164-168.
2. McDonald CJ, Hui SL, Smith DM, et al: Reminders to physicians from an introspective computer medical record: A two-year randomized trial Ann Intern Med 1984;100:130-138.

3. McDonald CJ: Protocol-based computer reminders: The quality of care and the non-perfectability of man. N Engl J Med 1976;295:1351-1355.

4. Jorgens J, Schneider RH: Regulation of medical device software: Role of FDA. Proceedings of AAMSI Congress 1986, May 1986, Anaheim, Calif, pp 43-46.

5. McDonald CJ: Computer-stored medical records: Their time is nigh, editorial. M.D. Comput 1987;4:7-8.

6. Cruickshank PJ: Patient satisfaction: Is the computer a plus or a minus? Update: Computer Med 1983;1(Sept-Oct):42-47.

7. Lloyd SC: Cost benefit analysis of Duchess in a primary care practice. AAMSI, October 1983.

8. Centers for Disease Control: United States Immunization Survey. Atlanta, Department of Health, Education and Welfare, 1979, no 79-8221.

From the Department of Family Medicine, Ohio University College of Osteopathic Medicine, Athens, Ohio.

Reprint requests to Dr Olson, Family Practice Center, PO Box 310, 20 Susquehanna Trail, Shamokin Dam, PA 17876. 